Article

\title{
On Variable Reverse Power Flow-Part I: Active-Reactive Optimal Power Flow with Reactive Power of Wind Stations
}

\author{
Aouss Gabash * and Pu Li \\ Department of Simulation and Optimal Processes, Institute of Automation and Systems Engineering, \\ Ilmenau University of Technology, Ilmenau 98693, Germany; pu.li@tu-ilmenau.de \\ * Correspondence: aouss.gabash@tu-ilmenau.de; Tel.: +49-3677-69-2813; Fax: +49-3677-69-1434
}

Academic Editor: Rodolfo Araneo

Received: 23 November 2015; Accepted: 4 February 2016; Published: 23 February 2016

\begin{abstract}
It has recently been shown that using battery storage systems (BSSs) to provide reactive power provision in a medium-voltage (MV) active distribution network (ADN) with embedded wind stations (WSs) can lead to a huge amount of reverse power to an upstream transmission network (TN). However, unity power factors (PFs) of WSs were assumed in those studies to analyze the potential of BSSs. Therefore, in this paper (Part-I), we aim to further explore the pure reactive power potential of WSs (i.e., without BSSs) by investigating the issue of variable reverse power flow under different limits on PFs in an electricity market model. The main contributions of this work are summarized as follows: (1) Introducing the reactive power capability of WSs in the optimization model of the active-reactive optimal power flow (A-R-OPF) and highlighting the benefits/impacts under different limits on PFs. (2) Investigating the impacts of different agreements for variable reverse power flow on the operation of an ADN under different demand scenarios. (3) Derivation of the function of reactive energy losses in the grid with an equivalent- $\pi$ circuit and comparing its value with active energy losses. (4) Balancing the energy curtailment of wind generation, active-reactive energy losses in the grid and active-reactive energy import-export by a meter-based method. In Part-II, the potential of the developed model is studied through analyzing an electricity market model and a 41-bus network with different locations of WSs.
\end{abstract}

Keywords: active-reactive energy losses; variable reverse power flow; varying power factors (PFs); wind power

\section{Introduction}

Buy-back is well-known in electricity markets where utilities or customers are buying or selling electric energy in a designed energy marketplace [1]. However, this issue becomes more complex if renewable energies and/or storage systems are considered in connected power systems with bidirectional active and reactive power flows as seen in Figure 1 [2].

Besides the literature review given in [2], we mention some other recent studies apparent in the area of reactive power of renewable energies for power market clearing. It is to note that new researches have been recently focused on optimal reactive power flow in transmission or distribution systems or in between based on a more detailed model. In [3], e.g., the authors proposed an algorithm to minimize reactive power provision, transmission loss costs and transmission system voltage security margin by a four-stage multi-objective mathematical programming method to settle the reactive power market. The authors in [3] focused later in [4] on the stochastic reactive power market in the presence of volatility of wind power generation in a transmission system. This kind of stochastic reactive power from wind generators was also studied in [5] for a medium-voltage (MV) network. The works in [4,5] 
used a model for reactive power production from wind turbines based on a detailed induction machine equivalent circuit as presented in [6]. Other researchers [7], however, used a different model based on an electrical model of a wind turbine with a full scale converter. We note that reactive power contribution can also be modeled differently form photovoltaic [8] or energy storage systems [2]. From another point of view, not only active and reactive powers from renewable energies are important but also the modeling of demand in hybrid electricity markets $[9,10]$. Since considering "at once" all aspects and concerns in the power system shown in Figure 1 is prohibitive, our focus in this work will be on the power sub-system depicted in Figure 2.

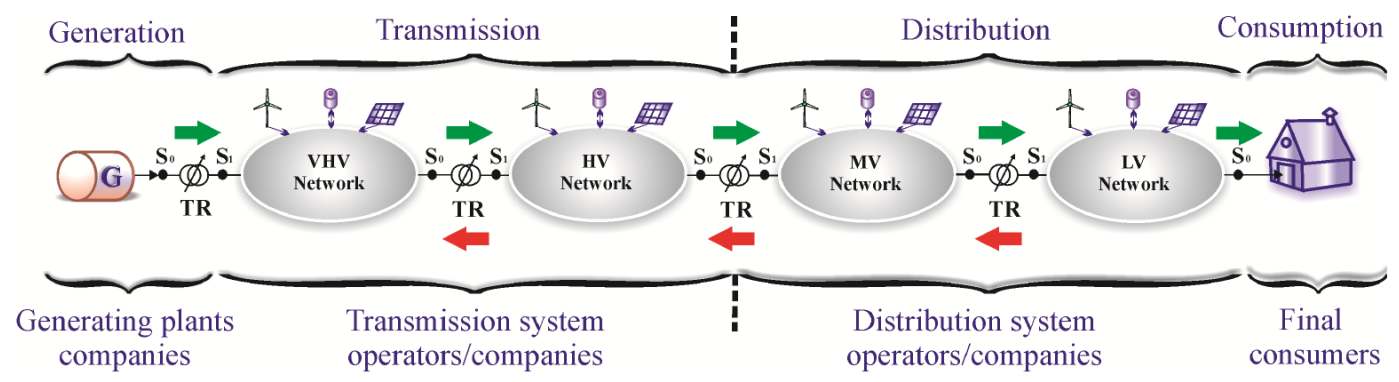

Figure 1. Conceptual structure of a future power system [2]. Here, $S_{0}$ stands for the primary side while $S_{1}$ for the secondary side of a transformer (TR) which is located between two different voltage levels.

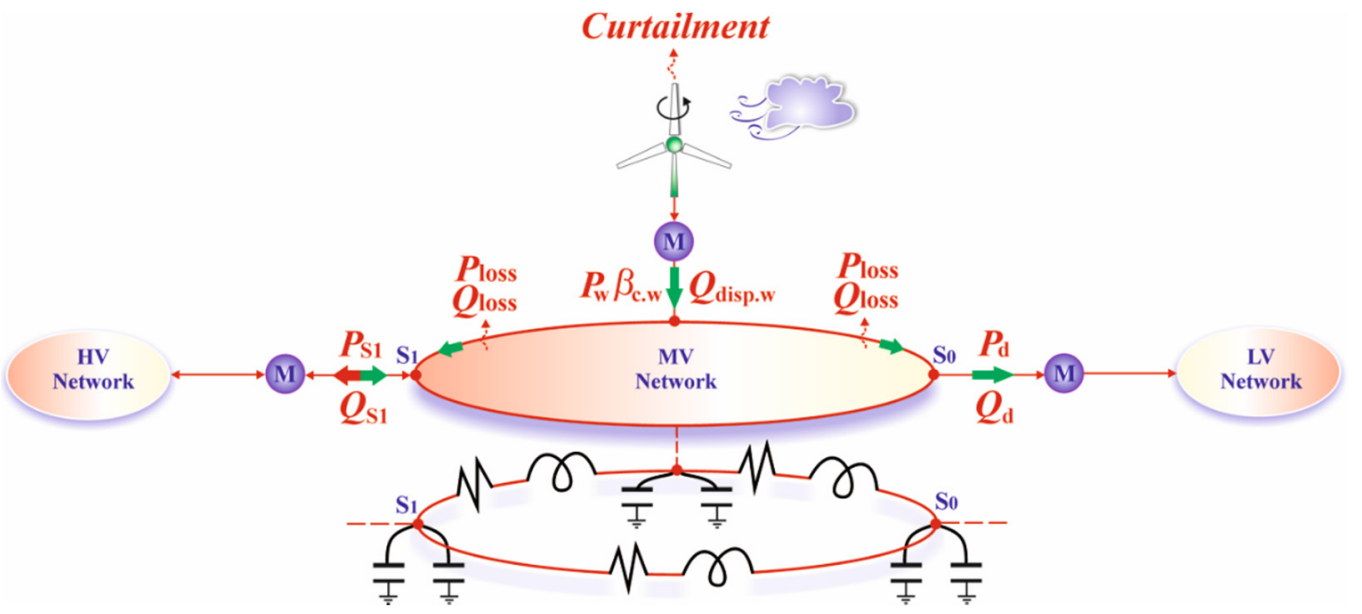

Figure 2. Illustration of the power system under consideration with a meter method [2]. In this paper, the low-voltage (LV) network is considered with unidirectional power flows while the high-voltage $(\mathrm{HV})$ network with bidirectional power flows.

As shown in Figure 2, at the high-voltage transmission network (HV-TN), firm [11] and non-firm [12] wind energy penetration can be maximized. It was mentioned in [13] that unit commitment issues and the stability of voltage at a HV-TN have high effects on wind energy losses. Based on [11-13], other important factors which influence wind energy losses in a HV-TN were studied in [14]. It was shown that the reverse active power flow from a downstream medium-voltage active distribution network (MV-ADN) could be rejected at the HV-TN level. Based on the initial analysis in [14], it was assumed in [15] and [16] that all rates of flows for active and reactive power to the HV-TN could be accepted. However, in $[15,16]$ wind stations (WSs) were assumed to operate with unity power factors (PFs) (i.e., $Q_{\text {disp.w }}=0$, see Figure 2 ) either for simplicity or to show the pure impacts of battery storage systems (BSSs).

Renewable energy curtailment and reverse power flow can also occur in a low-voltage ADN (LV-ADN) with a high renewable penetration from distributed generation (DG) units such as 
photovoltaic (PV) systems [17-19]. It was demonstrated in [19] that utilizing the reactive power capability of PV systems in a LV-ADN can lead to a huge amount of reactive energy import from an upstream connected MV network. This can lead to considerable active energy losses in the LV-ADN. Furthermore, it was mentioned that the costs of reactive energy to be purchased from a MV network is much cheaper than the active energy from PV systems. However, a balance between active and reactive energy prices, especially under reverse power flows, was not considered in previous studies.

In comparison to PV systems, wind DG units were also studied in [20] to provide reactive power and support medium voltage networks under an incremental loss allocation method. Nevertheless, it was assumed in [20] that wind DG units are able to provide reactive support via extra installed devices such as capacitor banks. However, installing any extra devices for providing reactive power provision leads to extra payments and consequently higher investment costs. It should be mentioned here that the power capability of DG units to provide active/reactive power highly depends on the technologies as shown in $[21,22]$. Briefly, utilizing the reactive power capability of DG units can significantly affect active energy losses in ADNs.

From another perspective, not only active energy losses in the grid are important in operating ADNs, but also the losses of reactive energy. This effect can be seen if a high price of reactive energy is considered, e.g., 90 \$/Mvarh [23]. Generally, energy prices can be assumed constant or being determined by an optimal contract price as in [24]. However, considering optimal contract prices needs to know the quantity of power generation and the time of dispatch. Therefore, such models are restricted to dispatchable DG technologies. For non-dispatchable DG units, e.g., those based on renewable energy generation, feed-in tariffs are applicable in different ways and countries [25].

In summary, the issue of metering bidirectional power [26] due to possible reverse active and reactive power flows [27-30] in a transformer (TR) [31] which is typically used to connect different voltage levels in power systems, as seen in Figure 1, is of high interest and major importance for the society of power and energy systems. This represents a combined technical and economical complex problem, and, therefore, more studies are required in this area. The main contributions of this work are summarized as follows:

- Introducing the reactive power capability of WSs in the optimization model of the active-reactive optimal power flow (A-R-OPF) and highlighting the benefits/impacts under different limits on PFs.

- Investigating the impacts of different agreements and variable reverse power flow on the operation of an ADN under different demand scenarios.

- Derivation of the function of reactive energy losses in the grid with an equivalent- $\pi$ circuit and comparing its value with active energy losses.

- Balancing the energy curtailment of wind generation, active/reactive energy losses in the grid and active/reactive energy import/export by a meter-based method.

The remainder of the paper is organized as follows: Section 2 describes the problem and introduces modeling procedures. In Section 3, the A-R-OPF model considering reactive power of WSs is developed. Section 4 concludes the paper.

\section{Problem Statement and Modeling Procedure}

Before formulating the A-R-OPF problem in a MV network, we would like to note that there are some major differences between optimal power flow problems in transmission and distribution systems. Such differences come from the fact that transmission systems are typically large and stretched on different areas with possibly dissimilar environmental issues. This leads to different system parameters and brings new technical and economic constraints in comparison to that in distribution systems. From another perspective, distribution systems could also include different entities which could be connected to balanced and/or unbalanced distribution networks. Therefore, a clear problem formulation should be given to distinguish such differences. For example, in Figure 1, if the focus is on the MV network, a 
model should be used to describe active/reactive power exchanges and voltage amplitudes/angles at the connection points, i.e., $S_{0}$ towards the LV network and $S_{1}$ towards the HV network. In addition, it should be clarified what exactly the MV network includes.

The authors in [15] analyzed the pure reactive power potential of BSSs in an ADN with WSs to maximize the revenues of wind power and BSSs and meanwhile minimize the costs of active energy losses. The WSs, however, were assumed to work with unity PFs. Therefore, it is aimed in this paper to further explore the reactive power potential of WSs "in the absence of BSSs" by considering variable reverse power flow and demand. In addition, we minimize the costs of both energy losses in the ADN, i.e., $P_{\text {loss }}$ and $Q_{\text {loss }}$. For clarity, a modeling procedure of each entity related to the balanced power system depicted in Figure 2 is described in the following.

\subsection{Wind Station}

Generally, the capability of a power conditioning system (PCS) used for connecting DG units to a power system depends on DG technologies, e.g., see [21]. In this paper, a full-scale power electronic converter [22] is modeled as an ideal PCS as seen in Figure 3. Furthermore, we incorporate a curtailment factor $\beta_{\text {c.w }}$ (see the nomenclature in Appendix 1) to ensure feasible solutions of the A-R-OPF as defined in [15] and [19]. The relationships between the active $P_{\mathrm{w}}$ and reactive $Q_{\text {disp.w }}$ power in this model are simply explained using Figure 3 and expressed as:

$$
\begin{gathered}
S_{\text {PCS.w }}(l, h)=\left(\left(P_{\mathrm{w}}(l, h) \beta_{\text {c.w }}(l, h)\right)^{2}+\left(Q_{\text {disp.w }}(l, h)\right)^{2}\right)^{0.5} \\
Q_{\text {disp.ava.w }}(l, h)= \pm\left(\left(S_{\text {PCS.max.w }}(l)\right)^{2}-\left(P_{\mathrm{w}}(l, h) \beta_{\text {c.w }}(l, h)\right)^{2}\right)^{0.5}
\end{gathered}
$$

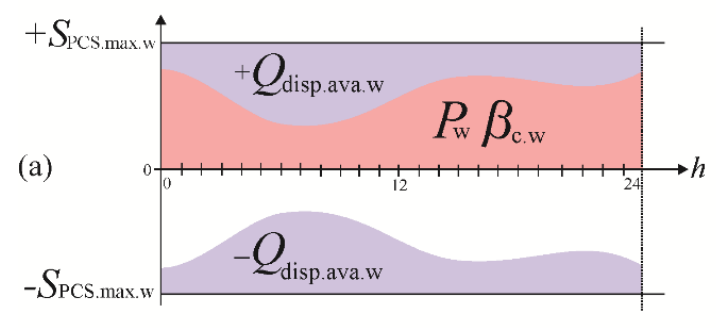

(b)

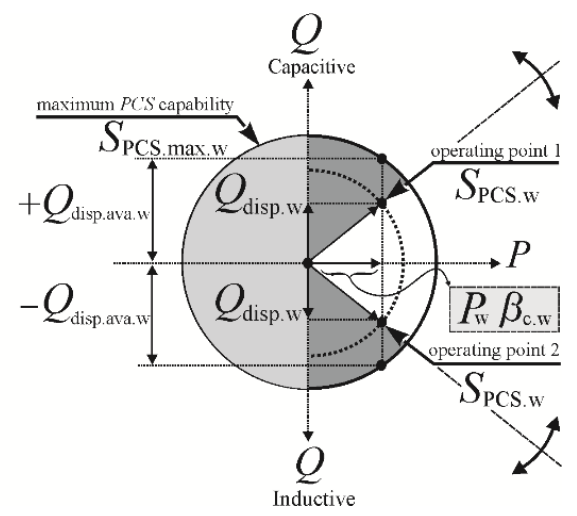

Figure 3. (a) Illustration of the behaviors of active/reactive powers through an ideal power conditioning system (PCS) [2]; and (b) the capability diagram of a PCS [2,22], where the dark-area stands for varying limits on power factors (PFs).

Here, the model used for transforming wind speed to wind power is considered as in [15]. The introduction of a curtailment factor $\left(0 \leqslant \beta_{\mathrm{c} . \mathrm{w}} \leqslant 1\right)$ at each WS is necessary to curtail the wind power generation to prevent violations of system constraints. More importantly, we consider the situation in which the available reactive power need to be restricted by different limits on PFs [21,22] (Figure 3a). This is represented with the dark-area in Figure 3b. Therefore, to study the impacts/benefits of such varying limits on PFs, additional constraints are introduced to the original model [19]:

$$
\begin{gathered}
S_{\text {PCS.w }}(l, h) \leqslant\left(S_{\text {PCS.max.w }}(l)=P_{\mathrm{W}}(l)\right) \\
\operatorname{PF}_{\text {min.w }}(l) S_{\text {PCS.w }}(l, h)-P_{\mathrm{w}}(l, h) \beta_{\text {c.w }}(l, h) \leqslant 0
\end{gathered}
$$




$$
\operatorname{PF}_{\text {max.w }}(l) S_{\text {PCS.w }}(l, h)-P_{\mathrm{w}}(l, h) \beta_{\text {c.w }}(l, h) \geqslant 0
$$

Here, $P F_{\max . w}=1$ represents the highest PF. In this study, we are concerned about the benefits/impacts of considering different values of $P F_{\text {min.w. }}$. In other words, $P F_{\text {min.w }}=1$ represents the original A-R-OPF model [15], otherwise specified.

\subsection{Substation Transformer}

Typically, distribution companies purchase active and reactive power from an external grid/market within the transfer limit of its substation TRs [23]. As depicted in Figure 1, a downstream network is being supplied by an upstream network via a TR which allows the flow of powers in two directions. For the MV network, the forward direction means import while the reverse direction means export [26-30]. Basically, the apparent power at bus $S_{1}$ is described as:

$$
S_{\mathrm{S} 1}(h)=\left(P_{\mathrm{S} 1}^{2}(h)+Q_{\mathrm{S} 1}^{2}(h)\right)^{0.5}
$$

In addition, it is assumed that the TR is equipped with an on-load tap changer control system which is typically required to hold the voltage amplitude at bus $S_{1}$ at a specific level. This can be achieved if a suitable number of taps is available [31]. The TR capacity is constrained in this paper by:

$$
\begin{gathered}
S_{\mathrm{S} 1}(h) \leqslant S_{\mathrm{S} 1 \text { max }} \\
-\alpha_{\mathrm{P} 1 \text {.rev }} S_{\mathrm{S} 1 \text { max }} \leqslant P_{\mathrm{S} 1}(h) \leqslant \alpha_{\mathrm{P} 1 . \mathrm{fw}} S_{\mathrm{S} 1 \text { max }} \\
-\alpha_{\mathrm{Q} 1 \text {.rev }} S_{\mathrm{S} 1 \text { max }} \leqslant Q_{\mathrm{S} 1}(h) \leqslant \alpha_{\mathrm{Q} 1 . \mathrm{fw}} S_{\mathrm{S} 1 \text { max }}
\end{gathered}
$$

Note that in [2], $\left(0 \leqslant \alpha_{\mathrm{P} 1 . f \mathrm{f}}, \alpha_{\mathrm{Q} 1 . f w}, \alpha_{\mathrm{P} 1 . \text { rev }}, \alpha_{\mathrm{Q} 1 . \mathrm{rev}} \leqslant 1\right)$ are considered as four decision variables with fixed values (i.e., $\alpha_{\mathrm{P} 1 . \mathrm{fw}}=1, \alpha_{\mathrm{Q} 1 . \mathrm{fw}}=1, \alpha_{\mathrm{P} 1 \text {.rev }}=0.6, \alpha_{\mathrm{Q} 1 \text {.rev }}=0.6$ ) to control the power flow rate at bus $S_{1}$ in two directions. However, using fixed values 0.6 (i.e., $60 \%$ of TR capacity) cannot reflect the whole effect of reverse power flow. The latter will possibly represent different agreements between the upstream and downstream networks shown in Figure 1. In addition, these decision variables represent the degree or level of freedom of transferring power at bus $S_{1}$ as depicted in Figure 4 where five possible operating states of an $\mathrm{ADN}$ are illustrated.

- State 1: This case represents an isolated power system where no active-reactive power is allowed to exchange between the upstream and downstream network at bus $S_{1}$. In this case, renewable/conventional generating units and/or BSSs should be able to satisfy the active-reactive demand and losses inside the downstream network.

- State 2: Only active power is allowed to be imported (purchased) from the upstream network to satisfy the demand and losses in the downstream network. In this case, reactive power should be generated locally by suitable means to satisfy the reactive demand and losses in the downstream network.

- State 3: This case can be considered as the conventional state where powers are purchased from the network under a high voltage level. The purchased power must satisfy the active/reactive demand and losses in the network under a low voltage level. Note that in all states 1-3 renewable/conventional generating units and/or BSSs can be integrated in the downstream network, but no reverse active-reactive power is allowed to be sold to the upstream network.

- State 4: Active/reactive power is allowed to be purchased from the upstream network to satisfy all or part of the active/reactive demand and losses in the downstream network, but only reverse active power is allowed to be sold to the upstream network. In comparison to the states 1-3, additional agreements between the two sides should be declared for allowing bidirectional active power flows at bus $S_{1}$. 
- State 5: Both active and reactive powers are allowed to exchange between the upstream and downstream network. Here, more agreements are required before allowing bidirectional active-reactive power flows at bus $S_{1}$.

The operating state can be denoted by the values of the four decision variables. For example, the state $(1,1$, variable, 0$)$ means $\left(\alpha_{\mathrm{P} 1 . \mathrm{fw}}=1, \alpha_{\mathrm{Q} 1 . \mathrm{fw}}=1, \alpha_{\mathrm{P} 1 . \mathrm{rev}}=\right.$ variable, $\left.\alpha_{\mathrm{Q} 1 . \mathrm{rev}}=0\right)$.

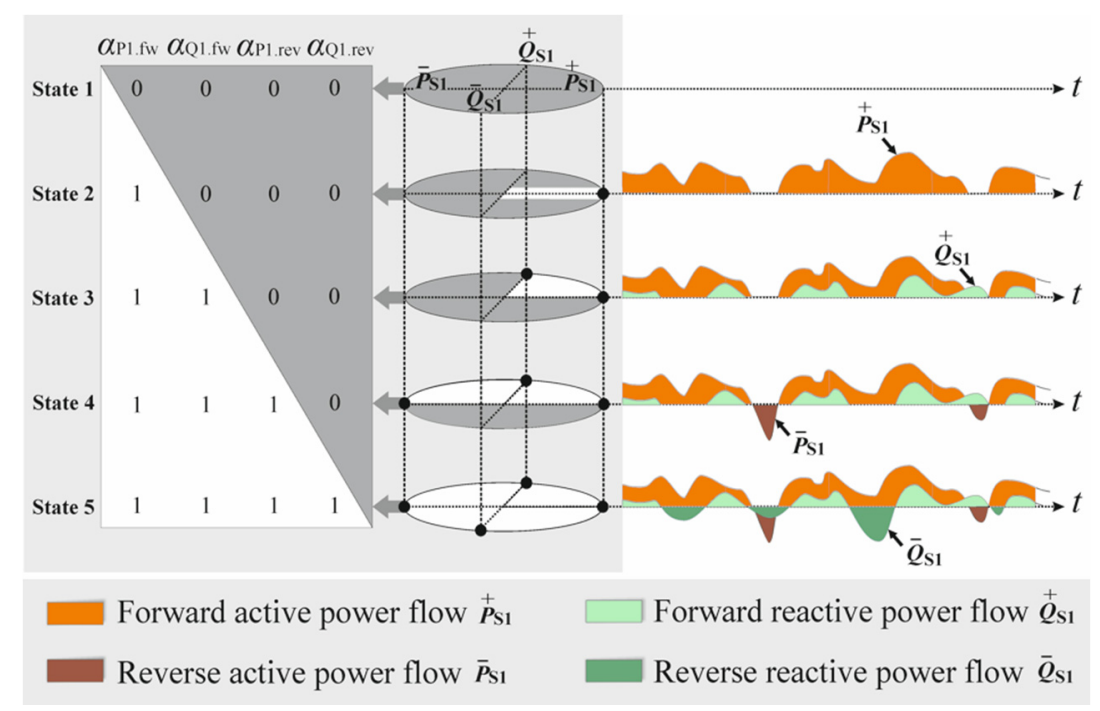

Figure 4. Conceptual illustration of the main five operating states. Here, the signs " +" /"-" mean import/export active/reactive power at a slack bus $S_{1}$, respectively, as defined in [2].

\subsection{Demand}

Based on the initial analysis in [14], active and reactive power demand from passive distribution networks (PDNs) and/or ADNs depend on possible internal optimal operations of both PDNs and ADNs. It means that the active and reactive power demand of the LV networks (see Figure 1) could be zero, positive and/or negative if a kind of power producer (e.g., renewable generators and/or storage systems) is embedded. In this work, for simplicity and clarity, both active and reactive demand power profiles are assumed to follow the Institute of Electrical and Electronics Engineers (IEEE) typical positive power demand in winter days [2]. Such demand is referred to as the base demand scenario $(100 \%)$. However, other different demand scenarios are also considered. Briefly, four demand scenarios, namely, very low (10\%), low (50\%), medium (100\%) and high (150\%) are considered and their impacts are analyzed.

\section{Active-Reactive Optimal Power Flow with Reactive Power of Wind Stations}

Typically, using different energy prices in any power marketplace affects the results significantly as shown in [16,19]. Therefore, for clear analysis, fixed tariff price models for both active and reactive energies $[15,16]$ are assumed in this work.

\subsection{Objective Function}

Based on Figure 2, the objective function of A-R-OPF considered in this paper is defined as Equation (10). The control variables in the proposed model of A-R-OPF are the curtailment factor of active power dispatch of WSs and the reactive power dispatch of WSs. It is aimed to maximize the revenue from the wind power Equation (11) and meanwhile minimizing the costs of both Equations (12) and (13) energy losses (see mathematical derivations in Appendix 2) in the MV network. 
In addition, we explicitly evaluate the costs of active and reactive energy (if being imported/purchased) in the Equations (14) and (15) or revenues (if being exported/sold) of only the active energy at bus $S_{1}$.

$$
\max _{\beta_{\text {c.w. }}, Q_{\text {disp.w }}} F=F_{1}-F_{2}-F_{3}-F_{4}-F_{5}
$$

where

$$
\begin{gathered}
F_{1}=\sum_{h=1}^{T_{\text {final }}} C_{\text {pr.p }}(h) \sum_{\substack{i=1 \\
i \in l}}^{N} P_{\mathrm{w}}(i, h) \beta_{\mathrm{c.w}}(i, h) \\
F_{2}=\sum_{h=1}^{T_{\text {final }}} C_{\text {pr.p }}(h) P_{\text {loss }}(h) \\
F_{3}=\sum_{h=1}^{T_{\text {final }}} C_{\text {pr.q }}(h) Q_{\text {loss }}(h) \\
F_{4}=\sum_{h=1}^{T_{\text {final }}} C_{\text {pr.p }}(h) P_{\mathrm{S} 1}(1, h) \\
F_{5}=\sum_{h=1}^{T_{\text {final }}} C_{\text {pr.q }}(h) Q_{\mathrm{S} 1}(1, h)
\end{gathered}
$$

\subsection{Equality Equations}

The active power flow equations considered in this paper are:

$$
\begin{aligned}
V_{\mathrm{e}}(i, h) \sum_{\substack{j=1 \\
j \in i}}^{N}\left(G(i, j) V_{\mathrm{e}}(j, h)-B(i, j) V_{\mathrm{f}}(j, h)\right) & +V_{\mathrm{f}}(i, h) \sum_{\substack{j=1 \\
j \in i}}^{N}\left(G(i, j) V_{\mathrm{f}}(j, h)+B(i, j) V_{\mathrm{e}}(j, h)\right) \\
& +P_{\mathrm{d}}(i, h)-P_{\mathrm{w}}(i, h) \beta_{\mathrm{c.w}}(i, h)-P_{\mathrm{S} 1}(1, h)=0, \quad i \in N
\end{aligned}
$$

while the reactive power flow equations are expressed as:

$$
\begin{aligned}
V_{\mathrm{f}}(i, h) \sum_{\substack{j=1 \\
j \in i}}^{N}\left(G(i, j) V_{\mathrm{e}}(j, h)-B(i, j) V_{\mathrm{f}}(j, h)\right) & -V_{\mathrm{e}}(i, h) \sum_{\substack{j=1 \\
j \in i}}^{N}\left(G(i, j) V_{\mathrm{f}}(j, h)+B(i, j) V_{\mathrm{e}}(j, h)\right) \\
& +Q_{\mathrm{d}}(i, h)-Q_{\text {disp.w }}(i, h)-Q_{\mathrm{s} 1}(1, h)=0, \quad i \in N .
\end{aligned}
$$

In order to show the contribution of WSs as the sole reactive power source in the MV network, no BSS is considered in this paper in comparison to [2]. More precisely, the MV network depicted in Figure 2 includes, as mathematically formulated in Equations (16) and (17), demand $\left(P_{\mathrm{d}}, Q_{\mathrm{d}}\right)$, WS $\left(P_{\mathrm{w}}, Q_{\text {disp.w }}\right)$ and slack bus $S_{1}\left(P_{\mathrm{S} 1}, Q_{\mathrm{S} 1}\right)$.

\subsection{Inequality Equations}

The inequality equations include the constraints of the voltage limits:

$$
V_{\min }(i) \leqslant V(i, h) \leqslant V_{\max }(i), i \in N\left(i \neq \mathrm{S}_{1}\right)
$$

and limits of distribution lines:

$$
S(i, j, h) \leqslant S_{1 \cdot \max }(i, j), i, j \in N(i \neq j)
$$


as well as the limits of the curtailment factors:

$$
0 \leqslant \beta_{\text {c.w }}(l, h) \leqslant 1
$$

In addition, and distinguished from the original A-R-OPF [15,16], the upper limits of apparent power (3) and PFs of WSs (4) and (5) as well as upper limits of apparent power at bus $S_{1}$ (7)-(9) are also included into the inequality constraints. It is to note, however, that stability constraints are not included in the above model and the formulated A-R-OPF problem is solved by the same general algebraic modeling system (GAMS) using the nonlinear programming (NLP) solver CONOPT3 as in $[15,16]$.

\subsection{Operating Conditions}

Based on the market strategies introduced in [15,16,23-25], we consider the following operating conditions:

- The operator of the MV network shown in Figure 2 is considered to be the sole owner of the network [24], who aims at a reliable operation of the system.

- The system operator aims to maximize the benefits from wind power and meanwhile to minimize the costs of both active and reactive energy losses [23].

- The imported/exported active energy from/to the upstream HV network, the active energy of WSs in the MV network and active energy losses in the MV network are calculated by a given price model for active energy, i.e., the fixed on-peak (100 \$/MWh) and off-peak (50\$/MWh) tariff price $[15,25]$.

- The imported/exported reactive energy and reactive energy losses in the MV network are calculated by a given price model, i.e., the fixed (12\$/Mvarh) tariff price [16].

- The reverse active energy to the HV network is permitted under different levels, while reverse reactive energy is not permitted for the reasons given in $[32,33]$.

\subsection{Questions}

From the above extended version of the A-R-OPF model, Part-II [33] will answer the following questions:

(1) What are the benefits/impacts of the "extended A-R-OPF model" for a real case power network?

(2) What are the benefits/impacts of "varying PFs" of WSs in days with different levels of wind power generation?

(3) How do the revenues/costs change with "variable reverse power flow" and "demand level" in an electricity market?

(4) What are the relationships between the "location" of WSs, "variable reverse power flow" and "feeder congestion"?

\section{Conclusions}

In this paper, the optimization model of A-R-OPF in ADNs is further explored by considering the reactive power capability of WSs. It is aimed to evaluate the potential/effects of considering varying PFs in the A-R-OPF model. It is also aimed to minimize not only active but also reactive energy losses in grids. This is done by extending the objective function and considering both active and reactive energy prices. Furthermore, the impact of "long-to-short" and "short-to-long" feeders for power system networks is also investigated. The relationships and the interplay between wind generation curtailment, variable reverse active power flows, varying PFs of WSs, different demand levels and active/reactive energy prices in an electricity market model are shown in Part-II of this paper. The developed model in Part-I and investigated case studies in Part-II will help power system planers avoid extra size of devices (e.g., batteries) for reactive power provision if WSs are available in ADNs. 
Moreover, the relationships between the "location" of WSs, "variable reverse power flow" and "feeder congestion" are also studied in Part-II.

Acknowledgments: We acknowledge support for the Article Processing Charge by the German Research Foundation and the Open Access Publication Fund of the Technische Universität Ilmenau.

Author Contributions: The corresponding author (Aouss Gabash) developed the model, wrote the code and performed the optimization; both authors (Aouss Gabash and P. Li) wrote the paper.

Conflicts of Interest: The authors declare no conflict of interest.

\section{Appendix 1}

The notation used throughout the paper is given below.

\section{Functions}

F

$F_{1}$

$F_{2}$

$F_{3}$

$F_{4}$

$F_{5}$

Parameters

$B(i, j)$

$B_{\text {se }}(i, j)$

$B_{\mathrm{sh}}(i, j)$

$C_{\text {pr.p }}(h)$

$C_{\text {pr.q }}(h)$

$G(i, j)$

$N$

$P_{\mathrm{d}}(i, h)$

$P_{\mathrm{W}}(l)$

$P_{\mathrm{w}}(i, h)$

$Q_{\mathrm{d}}(i, h)$

$S_{\text {l.max }}(i, j)$

$S_{\text {S1.max }}$

$S_{\text {PCS.max.w }}(l)$

$T_{\text {final }}$

$V_{\min } / V_{\max }(i)$

State Variables

$P_{\text {loss }} / Q_{\text {loss }}(h)$

$P_{\mathrm{S} 1} / Q_{\mathrm{S} 1}(1, h)$

$S(i, j, h)$

$S_{\text {PCS.w }}(l, h)$

$Q_{\text {dis.ava } \cdot w}(l, h)$

$V_{\mathrm{e}} / V_{\mathrm{f}}(i, h)$

$V(i, h)$

Control/Decision Variables

$Q_{\text {disp.w }}(l, h)$

$\beta_{\text {c.w }}(l, h)$

$\operatorname{PF}_{\text {min.w }}(l)$

$\operatorname{PF}_{\text {max.w }}(l)$

$\alpha_{\mathrm{P} 1 . f \mathrm{w}}$

$\alpha_{\mathrm{Q} 1 . f w}$

$\alpha_{P 1 \text {.rev }}$

$\alpha_{\mathrm{Q} 1 . r e v}$
Value of the objective function.

Revenue from wind power generation.

Cost of active energy losses.

Cost of reactive energy losses.

Cost/revenue of active energy at slack bus.

Cost/revenue of reactive energy at slack bus.

Imaginary part of the complex admittance matrix.

Imaginary part of the complex admittance matrix (series).

Imaginary part of the complex admittance matrix (shunt).

Price for active energy in hour $h$.

Price for reactive energy in hour $h$.

Real part of the complex admittance matrix.

Number of buses.

Power demand (active power) at bus $i$ in hour $h$.

Rated installed power of wind station (WS) $l$.

Wind power generation (active power) of WS at bus $i$ in hour $h$.

Power demand (reactive power) at bus $i$ in hour $h$.

Upper limit of apparent power for feeder located between bus $i$ and $j$.

Upper limit of apparent power at slack bus.

Upper limit of apparent power of WS $l$.

Time horizon.

Lower/upper limit of voltage amplitude at bus $i$.

Active/reactive power losses in hour $h$.

Active/reactive power produced/absorbed at slack bus in hour $h$.

Apparent power flow from bus $i$ to bus $j$ in hour $h$.

Apparent power of WS $l$ in hour $h$.

Available reactive power of WS $l$ in hour $h$.

Real/imaginary part of the complex voltage at bus $i$ in hour $h$.

Voltage amplitude at bus $i$ in hour $h$.

Reactive power dispatch of a WS $l$ in hour $h$.

Curtailment factor of wind power at WS $l$ during hour $h$.

Lower power factor of WS $l$.

Upper power factor of WS $l$.

Forward-limit on active power at bus $S_{1}$.

Forward-limit on reactive power at bus $S_{1}$.

Reverse-limit on active power at bus $S_{1}$.

Reverse-limit on reactive power at bus $S_{1}$. 


\section{Appendix 2}

In addition to the active power losses Equation (I) [15], reactive power losses can also be calculated for a given power network during hour $h$ as Equation (II):

$$
\begin{aligned}
P_{\text {loss }}(h)=\frac{1}{2} \sum_{i=1}^{N} \sum_{j=1}^{N} G(i, j)\left(V_{\mathrm{e}}^{2}(i, h)\right. & +V_{\mathrm{f}}^{2}(i, h)+V_{\mathrm{e}}^{2}(j, h)+V_{\mathrm{f}}^{2}(j, h) \\
& \left.-2\left(V_{\mathrm{e}}(i, h) V_{\mathrm{e}}(j, h)+V_{\mathrm{f}}(i, h) V_{\mathrm{f}}(j, h)\right)\right) \\
Q_{\text {loss }}(h)=\frac{1}{2} \sum_{i=1}^{N} \sum_{j=1}^{N}\left(B _ { \mathrm { se } } ( i , j ) \left(V_{\mathrm{e}}^{2}(i, h) \quad\right.\right. & V_{\mathrm{f}}^{2}(i, h)+V_{\mathrm{e}}^{2}(j, h)+V_{\mathrm{f}}^{2}(j, h) \\
& \left.-2\left(V_{\mathrm{e}}(i, h) V_{\mathrm{e}}(j, h)+V_{\mathrm{f}}(i, h) V_{\mathrm{f}}(j, h)\right)\right) \\
& \left.-B_{\mathrm{sh}}(i, j)\left(V_{\mathrm{e}}^{2}(i, h)+V_{\mathrm{f}}^{2}(i, h)\right)\right) .
\end{aligned}
$$

Furthermore, it is also aimed to continue the work in [32] by showing the impact of "long-to-short" and "short-to-long" feeders when calculating the imaginary part of the complex admittance matrixes in Equation (II). Therefore, a 4-bus illustrative network with a single phase equivalent- $\pi$ circuit and one line type $L 1$ is depicted in Figure A1, while a real case 41-bus network with different lines is provided in Part-II [33]. Note that the length of lines or feeders can lead to convergence problems in power flow calculations in terms of "ill-conditioned" power systems [2]. Here, $R_{1}, X_{1}$ and $B_{1}$ are the line resistance, inductive reactance and capacitive susceptance, respectively [2]. Let the value of base voltage equals $(27.6 \mathrm{kV})$, the value of base power equals (10 MVA) and the network data in Table A1, it gives the following matrices Equations (III)-(V) in pu system. It is mentioning that $B_{\mathrm{sh}}$ is a diagonal matrix and it will be equal to a zero matrix if $B_{1}$ is neglected in comparison to $R_{1}$ and $X_{1}$. Assuming that the load at bus 4 equals $10 \mathrm{MW}$ with $\mathrm{PF}=0.85$ lagging and the voltage amplitude at the slack bus No. 1 is taken $1.03 \mathrm{pu}$ and zero angle, then the active/reactive power balance and apparent power flow are as given in Tables A2 and A3.

$$
\begin{gathered}
B=\left[\begin{array}{cccc}
-31.309396 & 31.310149 & 0 & 0 \\
31.310149 & -156582.06 & 156550.751 & 0 \\
0 & 156550.751 & -156582.06 & 31.310149 \\
0 & 0 & 31.310149 & -31.309396
\end{array}\right] \\
B_{\text {se }}=\left[\begin{array}{cccc}
-31.310149 & 31.310149 & 0 & 0 \\
31.310149 & -156582.061 & 156550.751 & 0 \\
0 & 156550.751 & -156582.061 & 31.310149 \\
0 & 0 & 31.310149 & -31.310149
\end{array}\right] \\
B_{\mathrm{sh}}=\left[\begin{array}{cccc}
0.0015059 & 0 & 0 & 0 \\
0 & 0.0015063 & 0 & 0 \\
0 & 0 & 0.0015063 & 0 \\
0 & 0 & 0 & 0.0015059
\end{array}\right] .
\end{gathered}
$$

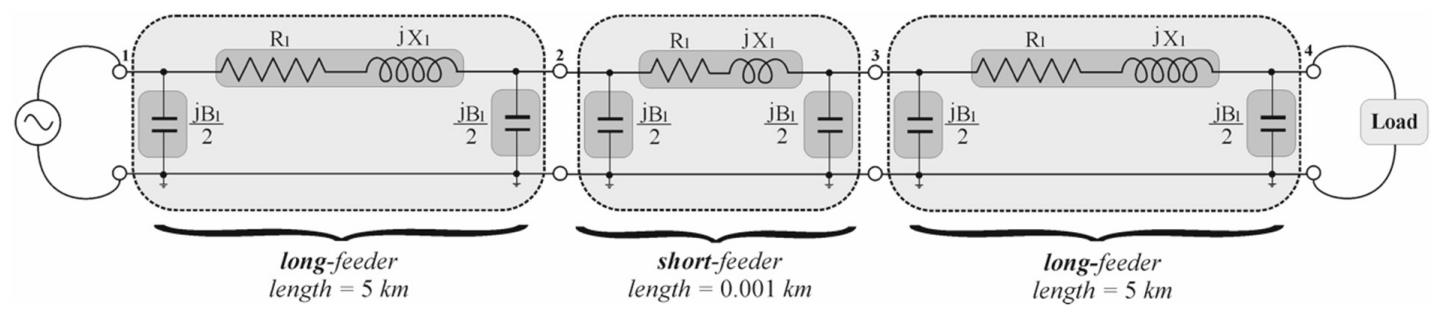

Figure A1. A single phase equivalent- $\pi$ circuit [2] of a 4-bus illustative network. Here, series and shunt parts of reactive power loss are considered. 
Table A1. Data of the ilustrative 4-bus network shown in Figure A1.

\begin{tabular}{|c|c|c|c|c|c|c|c|c|}
\hline No. Line & $\begin{array}{c}\text { From } \\
\text { Bus }\end{array}$ & $\begin{array}{c}\text { To } \\
\text { Bus }\end{array}$ & $\begin{array}{l}\text { Line } \\
\text { Type }\end{array}$ & $\begin{array}{c}\text { Length } \\
(\mathbf{k m})\end{array}$ & $\begin{array}{c}R_{1} \\
\text { (ohm/km) }\end{array}$ & $\begin{array}{c}X_{1} \\
\text { (ohm/km) }\end{array}$ & $\begin{array}{c}B_{1} \\
(\mu \mathrm{s} / \mathrm{km})\end{array}$ & $\begin{array}{l}\text { Ampacity } \\
\text { (MVA) }\end{array}$ \\
\hline 1 & 1 & 2 & $L 1$ & 5 & 0.169111 & 0.418206 & 3.954 & 20 \\
\hline 2 & 2 & 3 & $L 1$ & 0.001 & 0.169111 & 0.418206 & 3.954 & 20 \\
\hline 3 & 3 & 4 & $L 1$ & 5 & 0.169111 & 0.418206 & 3.954 & 20 \\
\hline
\end{tabular}

Table A2. Active and reactive power balance in the 4-bus network.

\begin{tabular}{cccccc}
\hline $\boldsymbol{P}_{\mathrm{d}}(\mathbf{M W})$ & $\boldsymbol{Q}_{\mathrm{d}}$ (Mvar) & $\boldsymbol{P}_{\text {loss }}(\mathbf{M W})$ & $Q_{\text {loss }}(\mathbf{M v a r})$ & $\boldsymbol{P}_{\mathrm{S} 1}(\mathbf{M W})$ & $Q_{\mathrm{S} 1}$ (Mvar) \\
\hline 10 & 6.2 & 0.33 & 0.77 & 10.33 & 6.97 \\
\hline
\end{tabular}

Table A3. Apparent power flow in the 4-bus network.

\begin{tabular}{ccc}
\hline$S(1,2)($ MVA) & $S(2,3)$ (MVA) & $S(3,4)($ MVA) \\
\hline 12.461 & 12.112 & 12.110 \\
\hline
\end{tabular}

\section{References}

1. Schweppe, F.C.; Caramanis, M.C.; Tabors, R.D.; Bohn, R.E. Spot Pricing of Electricity; Kluwer: Boston, MA, USA, 1988.

2. Gabash, A. Flexible Optimal Operations of Energy Supply Networks: With Renewable Energy Generation and Battery Storage; Südwestdeutscher Verlag: Saarbrücken, Germany, 2014.

3. Kargarian, A.; Raoofat, M.; Mohammadi, M. Reactive power market management considering voltage control area reserve and system security. Appl. Energy 2011, 88, 3832-3840. [CrossRef]

4. Kargarian, A.; Raoofat, M. Stochastic reactive power market with volatility of wind power considering voltage security. Energy 2011, 36, 2565-2571. [CrossRef]

5. Khorramdel, B.; Raoofat, M. Optimal stochastic reactive power scheduling in a microgrid considering voltage droop scheme of DGs and uncertainty of wind farms. Energy 2012, 45, 994-1006. [CrossRef]

6. Divya, K.C.; Nagendra Rao, P.S. Models for wind turbine generating systems and their application in load flow studies. Electr. Power Syst. Res. 2006, 76, 844-856. [CrossRef]

7. Samimi, A.; Kazemi, A.; Siano, P. Economic-environmental active and reactive power scheduling of modern distribution systems in presence of wind generations: A distribution market-based approach. Energy Convers. Manag. 2015, 106, 495-509. [CrossRef]

8. Aly, M.M.; Abdel-Akher, M.; Ziadi, Z.; Senjyu, T. Assessment of reactive power contribution of photovoltaic energy systems on voltage profile and stability of distribution systems. Electr. Power Energy Syst. 2014, 61, 665-672. [CrossRef]

9. Kargarian, A.; Raoofat, M.; Mohammadi, M. Probabilistic reactive power procurement in hybrid electricity markets with uncertain loads. Electr. Power Syst. Res. 2012, 82, 68-80. [CrossRef]

10. Haghighat, H.; Kennedy, S.W. A bilevel approach to operational decision making of a distribution company in competitive environments. IEEE Trans. Power Syst. 2012, 27, 1797-1807. [CrossRef]

11. Burke, D.J.; O'Malley, M.J. Maximizing firm wind connection to security constrained transmission networks. IEEE Trans. Power Syst. 2010, 25, 749-759. [CrossRef]

12. Burke, D.J.; O'Malley, M.J. A study of optimal nonfirm wind capacity connection to congested transmission systems. IEEE Trans. Sustain. Energy 2011, 2, 167-176. [CrossRef]

13. Burke, D.J.; O’Malley, M.J. Factors influencing wind energy curtailment. IEEE Trans. Sustain. Energy 2011, 2, 185-193. [CrossRef]

14. Gabash, A.; Xie, D.; Li, P. Analysis of influence factors on rejected active power from active distribution networks. In Proceedings of the IEEE Power and Energy Student Summit (PESS), Ilmenau, Germany, 19-20 January 2012; pp. 25-29. 
15. Gabash, A.; Li, P. Active-Reactive optimal power flow in distribution networks with embedded generation and battery storage. IEEE Trans. Power Syst. 2012, 27, 2026-2035. [CrossRef]

16. Gabash, A.; Li, P. Flexible optimal operation of battery storage systems for energy supply networks. IEEE Trans. Power Syst. 2013, 28, 2788-2797. [CrossRef]

17. Tonkoski, R.; Lopes, L.A.C.; El-Fouly, T.H.M. Coordinated active power curtailment of grid connected PV inverters for overvoltage prevention. IEEE Trans. Sustain. Energy 2011, 2, 139-147. [CrossRef]

18. Tonkoski, R.; Turcotte, D.; EL-Fouly, T.H.M. Impact of high PV penetration on voltage profiles in residential neighborhoods. IEEE Trans. Sustain. Energy 2012, 3, 518-527. [CrossRef]

19. Gabash, A.; Li, P. Active-Reactive optimal power flow for low-voltage networks with photovoltaic distributed generation. In Proceedings of the 2012 IEEE International Energy Conference and Exhibition (ENERGYCON), Florence, Italy, 9-12 September 2012; pp. 381-386.

20. Oliveira, P.M.D.; Jesus, P.M.; Castronuovo, E.D.; Leao, M.T. Reactive power response of wind generators under an incremental network-loss allocation approach. IEEE Trans. Energy Convers. 2008, 23, 612-621.

21. Zou, K.; Agalgaonkar, A.P.; Muttaqi, K.M.; Perera, S. Distribution system planning with incorporating DG reactive capability and system uncertainties. IEEE Trans. Sustain. Energy 2012, 3, 112-123. [CrossRef]

22. Ullah, N.R.; Bhattacharya, K.; Thiringer, T. Wind farms as reactive power ancillary service providers-technical and economic issues. IEEE Trans. Energy Convers. 2009, 24, 661-672. [CrossRef]

23. Algarni, A.A.S.; Bhattacharya, K. Disco operation considering DG units and their goodness factors. IEEE Trans. Power Syst. 2009, 24, 1831-1840. [CrossRef]

24. López-Lezama, J.M.; Padilha-Feltrin, A.; Contreras, J.; Muñoz, J.I. Optimal contract pricing of distributed generation in distribution networks. IEEE Trans. Power Syst. 2001, 26, 128-136. [CrossRef]

25. Mabeea, W.E.; Mannionb, J.; Carpenterc, T. Comparing the feed-in tariff incentives for renewable electricity in Ontario and Germany. Energy Policy 2012, 40, 480-489. [CrossRef]

26. Stevens, R.H. Power flow direction definitions for metering of bidirectional power. IEEE Trans. Power Appar. Syst. 1983, 9, 3018-3022. [CrossRef]

27. Levi, V.; Kay, M.; Povey, I. Reverse power flow capability of tap-changers. In Proceedings of the 18th International Conference Exhibition Electricity Distribution, Turin, Italy, 6-9 June 2005; pp. 1-5.

28. Cipcigan, L.M.; Taylor, P.C. Investigation of the reverse power flow requirements of high penetrations of small-scale embedded generation. IET Renew. Power Gener. 2007, 1, 160-166. [CrossRef]

29. Gabash, A.; Alkal, M.E.; Li, P. Impact of allowed reverse active power flow on planning PVs and BSSs in distribution networks considering demand and EVs growth. In Proceedings of the IEEE Power and Energy Student Summit (PESS), Bielefeld, Germany, 23-25 January 2013; pp. 11-16.

30. Gabash, A.; Li, P. Reverse active-reactive optimal power flow in ADNs: Technical and economical aspects. In Proceedings of the 2014 IEEE International Energy Conference (ENERGYCON), Cavtat, Croatia, 13-16 May 2014; pp. 1115-1120.

31. Gabash, A.; Li, P. On the control of main substations between transmission and distribution systems. In Proceedings of the 2014 14th International Conference on Environment and Electrical Engineering (EEEIC), Krakow, Poland, 10-12 May 2014; pp. 280-285.

32. Gabash, A.; Li, P. Variable reverse power flow-part I: A-R-OPF with reactive power of wind stations. In Proceedings of the 2015 IEEE 15th International Conference on Environment and Electrical Engineering (EEEIC), Rome, Italy, 10-13 June 2015; pp. 21-26.

33. Gabash, A.; Li, P. Variable reverse power flow-part II: Electricity market model and results. In Proceedings of the 2015 IEEE 15th International Conference on Environment and Electrical Engineering (EEEIC), Rome, Italy, 10-13 June 2015; pp. 27-32.

(C) 2016 by the authors; licensee MDPI, Basel, Switzerland. This article is an open access article distributed under the terms and conditions of the Creative Commons by Attribution (CC-BY) license (http://creativecommons.org/licenses/by/4.0/). 